\title{
Projectively and conformally invariant star-products
}

\author{
C. Duval ${ }^{\ddagger} \quad$ A.M. El Gradechi ${ }^{\S} \quad$ V. Ovsienko
}

\begin{abstract}
We consider the Poisson algebra $\mathcal{S}(M)$ of smooth functions on $T^{*} M$ which are fiberwise polynomial. In the case where $M$ is locally projectively (resp. conformally) flat, we seek the star-products on $\mathcal{S}(M)$ which are $\operatorname{SL}(n+1, \mathbb{R})$ (resp. $\mathrm{SO}(p+1, q+1)$ )-invariant. We prove the existence of such starproducts using the projectively (resp. conformally) equivariant quantization, then prove their uniqueness, and study their main properties. We finally give an explicit formula for the canonical projectively invariant star-product.
\end{abstract}

Keywords: Quantization, projective structures, conformal structures, star-product, invariant theory.

\section{Introduction}

The deformation quantization program initiated in the seventies [3] was aimed at defining an autonomous quantization method based on Gerstenhaber's general theory of deformation of algebraic structures [28]. The original idea was to view quantum mechanics as a one-parameter deformation of classical mechanics, more precisely, a one-parameter deformation of the algebraic structures underlying classical mechanics.

\footnotetext{
‡Université de la Méditerranée and CPT-CNRS, Luminy Case 907, F-13288 Marseille, Cedex 9, FRANCE; mailto:duval@cpt.univ-mrs.fr

${ }^{\S}$ Faculté des Sciences, Université d'Artois, F-62307 Lens, FRANCE and CPT-CNRS, Luminy Case 907, F-13288 Marseille, Cedex 9, FRANCE; mailto:amine@euler.univ-artois.fr

IInstitut Girard Desargues, Université Claude Bernard Lyon 1, F-69622 Villeubanne, Cedex, FRANCE; mailto:ovsienko@desargues.univ-lyon1.fr
} 
If $P$ is a Poisson manifold, then $C^{\infty}(P)$ is naturally equipped with two algebraic structures, namely, the associative and commutative pointwise multiplication and the Lie algebra defined by the Poisson bracket. The deformed algebraic structure, describing the quantum mechanical counterpart of $\left(C^{\infty}(P), \cdot,\{\cdot, \cdot\}\right)$ is $\left(C^{\infty}(P)[[\hbar]], \star\right)$, where the operation $\star$, called star-product, is an associative (but non-commutative) product on $C^{\infty}(P)[[\hbar]]$ deforming the commutative multiplication in the direction of the Poisson bracket. More precisely:

Definition 1.1. Let $P$ be a Poisson manifold and $C^{\infty}(P)$ the space of smooth complex-valued functions on $P$. A star-product on $P$ is an associative algebra structure on $C^{\infty}(P)[[\hbar]]$, denoted $\star$, and given by a linear map from $C^{\infty}(P) \otimes C^{\infty}(P)$ to $C^{\infty}(P)[[\hbar]]$, extended by linearity to $C^{\infty}(P)[[\hbar]] \otimes C^{\infty}(P)[[\hbar]]$, such that

$$
F \star G=F \cdot G+\frac{i \hbar}{2}\{F, G\}+\sum_{r=2}^{\infty}(i \hbar)^{r} B_{r}(F, G) .
$$

In the mathematical literature $\hbar$ is a formal parameter, whereas in physical applications $\hbar$ is Planck's constant.

There are usually three extra requirements for star-products :

C1. the constant function $\mathbf{1}$ is the unit of $\left(C^{\infty}(P)[[\hbar]], \star\right)$, namely $\mathbf{1} \star F=F \star \mathbf{1}=F$;

C2. the star-product is symmetric, viz $\overline{F \star G}=\bar{G} \star \bar{F}$;

C3. the bilinear maps $B_{r}$ are given by bidifferential operators.

Note that Condition $\mathrm{C} 2$ is sometimes called parity condition.

The first reported star-product appeared in the work of Gronewold [31. It was derived from the Weyl-Wigner quantization on $P=\mathbb{R}^{2 n}$. It is nowadays more commonly known as the Moyal star-product ; Moyal actually obtained the Lie algebra bracket associated with Grœenewold's star-product [39. This first star-product was later on rediscovered by Vey [44].

The general problem of existence of star-products was raised in [3]. Using cohomological techniques, De Wilde and Lecomte [16] proved the existence of starproducts on any symplectic manifold. A geometric proof of the same result together with an algorithmic construction was obtained by Fedosov [24, 25] (see [45] for a survey of this construction and [41] for an alternative approach). 
More recently, Kontsevich proved an existence theorem for an arbitrary Poisson manifold, giving explicit formulæ for $P=\mathbb{R}^{n}[34$. An operadic and a quantum field theoretic interpretations of Kontsevich's result were later on given respectively by Tamarkin [43, and Cattaneo and Felder [1].

The problem of the uniqueness of star-products is usually studied modulo equivalence (see Section 2.2 for definitions and [32, 14] for recent developments). However, extra conditions can sometimes be imposed to single out a canonical starproduct. For instance, Gutt 33 proved that the Moyal star-product is the unique $\left(\operatorname{Sp}(2 n, \mathbb{R}) \ltimes \mathbb{R}^{2 n}\right)$-invariant and covariant star-product on $\mathbb{R}^{2 n}$. The notion of a $\mathfrak{G}$-invariant star-product, where $\mathfrak{G}$ is a Lie group of Poisson automorphisms of $P$, was introduced in [3] (see Section 2.1 for definitions). Existence of a $\mathfrak{G}$-invariant star-product on a symplectic manifold was proved by Lichnerowicz 38 for any compact Lie group $\mathfrak{G}$ of symplectomorphisms. More recently, Fedosov [26] constructed a $\mathfrak{G}$-invariant star-product on a symplectic manifold endowed with a $\mathfrak{G}$-invariant symplectic connection.

In this article, we deal with cotangent bundles $P=T^{*} M$ equipped with their canonical symplectic structure, and restrict considerations to the Poisson algebra $\mathcal{S}(M)$ of smooth functions on $T^{*} M$ polynomial on fibers. We furthermore assume $M$ to be a smooth $n$-dimensional manifold endowed with either a projectively or a conformally flat structure, i.e., $M$ admits a (locally defined) action of either $\mathrm{SL}(n+1, \mathbb{R})$ or $\mathrm{SO}_{0}(p+1, q+1)$, the connected component of the pseudo-orthogonal group with $n=p+q$. The basic example of a projectively (resp. conformally) flat manifold is $\mathbb{R P}^{n}$ (resp. $\left.\left(S^{p} \times S^{q}\right) / \mathbb{Z}_{2}\right)$.

Denote by $\mathfrak{G}$ either the projective or the conformal group. We study, in the present article, $\mathfrak{G}$-invariant star-products on $T^{*} M$, where the $\mathfrak{G}$-action is the canonical lift of the natural action on the base. Our first result, Theorem 5.1, establishes the uniqueness of a $\mathfrak{G}$-invariant homogeneous star-product on $\mathcal{S}(M)$. Our second result, Theorem [5.7 proves the uniqueness of a $\mathfrak{G}$-invariant star-product modulo $\mathfrak{G}$-equivalence and reparametrization.

Let us emphasize that we do not assume conditions C1, C2 and C3 a priori. It turns out that $\mathrm{C} 1$ and $\mathrm{C} 2$ are automatically satisfied while C3 doesn't hold; in fact, the maps $B_{r}$ in (1.1) are pseudo-differential bilinear operators. Our $\mathfrak{G}$-invariant star-products cannot be obtained by Fedosov's or Kontsevich's constructions, as the 
latter lead to bidifferential star-products.

The existence of $\mathfrak{G}$-invariant star-products on $\mathcal{S}(M)$ is based on the existence of a $\mathfrak{G}$-equivariant quantization map [37, 23] (see also [22]). The latter is the unique (up to normalization) isomorphism of $\mathfrak{G}$-modules, $\mathcal{Q}_{\lambda}: \mathcal{S}(M) \rightarrow \mathcal{D}_{\lambda}(M)$, where $\mathcal{D}_{\lambda}(M)$ is the space of differential operators acting on tensor densities of degree $\lambda$. Such a quantization map defines a $\mathfrak{G}$-invariant associative product on $\mathcal{S}(M)$ which turns out to be a star-product for $\lambda=\frac{1}{2}$ as proved in [9, 23]. The existence and uniqueness results of the present article represent the deformation quantization counterparts of those obtained for $\mathfrak{G}$-equivariant quantization. In both situations invariance properties ensure uniqueness.

The pseudo-differential nature of the $\mathfrak{G}$-invariant star-products has been revealed by Brylinski [9] and Astashkevich and Brylinski [2]. In the latter Reference, invariant star-products on minimal nilpotent coadjoint orbits of semi-simple Lie groups have been investigated. These results are closely related to ours since these orbits are punctured cotangent bundles $T^{*} M \backslash M$; nevertheless the Poisson algebras considered in 2] are smaller than $\mathcal{S}(M)$. Moreover, our approach provides explicit formulæ in the projective case, answering a question raised in [2].

The paper is organized as follows. In Section 2 we recall the notions of invariant and equivalent star-products, and we give a short account on equivariant quantization for cotangent bundles. In Section 3, we define projective and conformal geometries and determine the ring of projectively/conformally invariant linear operators on $\mathcal{S}(M)$. The existence of $\mathfrak{G}$-invariant star-product on $T^{*} M$, along with a few of their properties, are proved in Section 4. Section 5 contains our uniqueness theorems. In Section 6, we give an autonomous derivation of the canonical projectively invariant star-product on $\mathcal{S}\left(\mathbb{R P}^{n}\right)$, based only on projective invariant theory. Explicit formulæ are then provided. We end this paper, with Section 7, where we gather our conclusion, a discussion and a few perspectives.

Acknowledgements: It is a pleasure to thank Ranee Brylinski, Simone Gutt, Pierre Lecomte and John Rawnsley for valuable help and encouragement. This work was done while the second author was visiting CPT as a délégué CNRS; he thanks CNRS for granting him a délégation and the Université d'Artois for consenting a one year leave of absence. The second and third authors both thank the CPT for hospitality. 


\section{Invariant star-products and equivariant quanti- zation}

In this section we introduce the general notions of invariance and covariance of star-products with respect to a Hamiltonian action of a connected Lie group $\mathfrak{G}$.

\subsection{Invariant, covariant and strongly invariant star-products}

First of all, let us give the precise definition of an invariant star-product already mentioned in the Introduction.

Definition 2.1. Given a Poisson action of a Lie group $\mathfrak{G}$ on a Poisson manifold $P$, a star-product $\star$ on $C^{\infty}(P)$ is called $\mathfrak{G}$-invariant if

$$
g^{*}(F \star G)=g^{*} F \star g^{*} G
$$

for all $F, G \in C^{\infty}(P)[[\hbar]]$ and $g \in \mathfrak{G}$.

In the case where the $\mathfrak{G}$-action is Hamiltonian one has the following supplementary notions.

Definition 2.2. Consider a Hamiltonian $\mathfrak{G}$-action on a Poisson manifold $P$ with associated equivariant moment map $J: P \rightarrow \mathfrak{g}^{*}$, where $\mathfrak{g}^{*}$ is the dual of the Lie algebra $\mathfrak{g}$ of $\mathfrak{G}$. A star-product on $P$ is called

a) $\mathfrak{G}$-covariant if

$$
J_{X} \star J_{Y}-J_{Y} \star J_{X}=i \hbar\left\{J_{X}, J_{Y}\right\}
$$

b) strongly $\mathfrak{G}$-invariant if

$$
J_{X} \star F-F \star J_{X}=i \hbar\left\{J_{X}, F\right\}
$$

for all $F \in C^{\infty}(P)[[\hbar]]$ and $X, Y \in \mathfrak{g}$, where $J_{X}$ is the Hamiltonian function on $P$ corresponding to $X$.

Remark 2.3. Note that a different terminology is sometimes attached to this last notion in the literature. What we call here strong $\mathfrak{G}$-invariance corresponds to the notion of preferred observables in [3, 18] and to Property $\mathrm{IP}_{2}$ in [1]. Beware that, in the latter Reference, strong invariance means covariance and invariance. 
Let us now recall the following useful result.

Proposition 2.4 ([1]). If a star-product is strongly $\mathfrak{G}$-invariant, then it is both $\mathfrak{G}$-invariant and $\mathfrak{G}$-covariant.

Proof. Using the definition (2.3) of strong invariance, we write

$$
\begin{aligned}
i \hbar\left\{J_{X}, F \star G\right\} & =J_{X} \star F \star G-F \star G \star J_{X} \\
& =J_{X} \star F \star G-F \star J_{X} \star G+F \star J_{X} \star G-F \star G \star J_{X} \\
& =i \hbar\left(\left\{J_{X}, F\right\} \star G+F \star\left\{J_{X}, G\right\}\right)
\end{aligned}
$$

which is nothing but the infinitesimal version of formula (2.1) expressing the invariance property. The $\mathfrak{G}$-invariance of the star-product then follows from the connectedness of $\mathfrak{G}$.

As for covariance, it is an immediate consequence of strong invariance.

Remark 2.5. The converse of Proposition 2.4 is proved in [1] under the additional assumption of a transitive $\mathfrak{G}$-action.

\subsection{Equivalence, $\mathfrak{G}$-equivalence and reparametrization}

In the traditional classification of star-products one introduces a notion of equivalence. Two star-products, $\star$ and $\star^{\prime}$, are called equivalent if there exists a formal series

$$
\Phi=\operatorname{Id}+i \hbar \Phi_{1}+(i \hbar)^{2} \Phi_{2}+\cdots
$$

where $\Phi_{i}: C^{\infty}(P) \rightarrow C^{\infty}(P)$ are some linear operators, such that

$$
\Phi(F) \star \Phi(G)=\Phi\left(F \star^{\prime} G\right) .
$$

Usually, one also allows for formal changes of the parameter of deformation:

$$
\mu: i \hbar \mapsto i \hbar+\sum_{k=2}^{\infty} a_{k}(i \hbar)^{k}
$$

where $a_{k} \in \mathbb{R}$, in order to comply with Property C2 from the Introduction. 
For $\mathfrak{G}$-invariant star-products it is natural to consider the notion of $\mathfrak{G}$-equivalence.

Definition 2.6 ([38]). Two equivalent $\mathfrak{G}$-invariant star-products are called $\mathfrak{G}$-equivalent if each map $\Phi_{i}$ in (2.4) is $\mathfrak{G}$-equivariant.

The condition for two star-products to be $\mathfrak{G}$-equivalent is much stronger than the usual condition of equivalence (see 4] for recent developments).

\subsection{Equivariant quantization and the associated invariant star-product}

Equivariant quantization as developed in [37, 23, 21, 22] applies to cotangent bundles. From here on we restrict ourselves to $P=T^{*} M$ endowed with its canonical symplectic form.

Let $\mathcal{S}(M) \subset C^{\infty}\left(T^{*} M\right)$ be the space of (complex-valued) functions on $T^{*} M$ polynomial on fibers, and $\mathcal{D}(M)$ be the space of linear differential operators acting on $C^{\infty}(M)$. The space $\mathcal{S}(M)$ is the space of symbols of operators in $\mathcal{D}(M)$; it has a natural grading

$$
\mathcal{S}(M)=\bigoplus_{k=0}^{\infty} \mathcal{S}_{k}(M)
$$

by the degree of homogeneity.

Let $\mathcal{F}_{\lambda}(M)$ be the space of tensor densities of degree $\lambda \in \mathbb{C}$ on $M$, i.e., the space of sections of the complex line bundle $\left|\Lambda^{n} T^{*} M\right|^{\lambda} \otimes \mathbb{C}$. In local coordinates such densities are of the form

$$
f\left(x^{1}, \ldots, x^{n}\right)\left|d x^{1} \wedge \cdots \wedge d x^{n}\right|^{\lambda}
$$

with $f \in C^{\infty}(M)$. Denote $\mathcal{D}_{\lambda}(M)$ the space of linear differential operators on $\mathcal{F}_{\lambda}(M)$; it has a natural filtration

$$
\mathcal{D}_{\lambda}^{0}(M) \subset \mathcal{D}_{\lambda}^{1}(M) \subset \cdots \subset \mathcal{D}_{\lambda}^{k}(M) \subset \cdots
$$

such that $\mathcal{S}(M)=\operatorname{gr}\left(\mathcal{D}_{\lambda}(M)\right)$.

Definition 2.7. A quantization map is an invertible linear map

$$
\mathcal{Q}_{\lambda}: \mathcal{S}(M) \rightarrow \mathcal{D}_{\lambda}(M)[\hbar]
$$


which preserves the principal symbol in the following sense : for a homogeneous polynomial $F \in \mathcal{S}_{k}(M)$, the principal symbol of the differential operator $\mathcal{Q}_{\lambda}(F)$ is equal to $(i \hbar)^{k} F$.

There is a natural action of the group of diffeomorphisms, $\operatorname{Diff}(M)$, on $\mathcal{F}_{\lambda}(M)$, denoted by $g_{\lambda}: \mathcal{F}_{\lambda}(M) \rightarrow \mathcal{F}_{\lambda}(M)$ for all $g \in \operatorname{Diff}(M)$. We will rather use the corresponding action of the Lie algebra of vector fields, $\operatorname{Vect}(M)$, which is given by

$$
L_{X}^{\lambda} f=X^{i} \frac{\partial f}{\partial x^{i}}+\lambda \frac{\partial X^{i}}{\partial x^{i}} f
$$

for all $X=X^{i} \partial / \partial x^{i} \in \operatorname{Vect}(M)$, with the local identification $\mathcal{F}_{\lambda}(M) \cong C^{\infty}(M)$ made in (2.8). (We will use Einstein's summation convention throughout this article.) Note that the expression (2.9) is, indeed, independent of the choice of a coordinate system. The canonical lift of the $\operatorname{Diff}(M)$-action to $T^{*} M$ is automatically Hamiltonian with moment map $J$ given by

$$
J_{X}=\xi_{i} X^{i} \in \mathcal{S}_{1}(M)
$$

Definition 2.8. Consider a Lie group $\mathfrak{G} \subset \operatorname{Diff}(M)$. A quantization map $\mathcal{Q}_{\lambda}$ is called $\mathfrak{G}$-equivariant if

$$
\mathcal{Q}_{\lambda}\left(g^{*} F\right)=g_{\lambda}^{-1} \circ \mathcal{Q}_{\lambda}(F) \circ g_{\lambda}
$$

for all $g \in \mathfrak{G}$ and $F \in \mathcal{S}(M)$.

The above formula plays a central rôle in the forthcoming developments. We will need its infinitesimal guise

$$
L_{X}^{\lambda} \circ \mathcal{Q}_{\lambda}(F)-\mathcal{Q}_{\lambda}(F) \circ L_{X}^{\lambda}=Q_{\lambda}\left(L_{X} F\right)
$$

for all $X \in \mathfrak{g}$, where $L_{X}$ stands for the canonical lift to $T^{*} M$ of the fundamental vector field associated with $X$.

From such a quantization map, we immediately obtain an associative product given by

$$
F \star_{\lambda} G=\mathcal{Q}_{\lambda}^{-1}\left(\mathcal{Q}_{\lambda}(F) \circ \mathcal{Q}_{\lambda}(G)\right) .
$$

Note that this product is not necessarily of the form (1.1). However, Condition C1 is automatically satisfied.

The following proposition is a direct consequence of the above definitions. 
Proposition 2.9. If $\mathcal{Q}_{\lambda}$ is a $\mathfrak{G}$-equivariant quantization map on $\mathcal{S}(M)$, then the associative product on $\mathcal{S}(M)$ given by (2.13) is $\mathfrak{G}$-invariant.

One wonders if there exists some extra condition sufficient to insure strong $\mathfrak{G}$-invariance of the $\mathfrak{G}$-invariant associative product (2.13). The next proposition introduces a natural geometric property of the quantization map that leads to the desired result.

Proposition 2.10. If $\mathcal{Q}_{\lambda}$ is a $\mathfrak{G}$-equivariant quantization map on $\mathcal{S}(M)$, which furthermore satisfies the following condition

$$
\mathcal{Q}_{\lambda}\left(J_{X}\right)=i \hbar L_{X}^{\lambda}
$$

for all $X \in \mathfrak{g}$, then the associative product on $\mathcal{S}(M)$ given by (2.13) is strongly $\mathfrak{G}$-invariant .

Proof. Let $X \in \mathfrak{g}$ and $F \in \mathcal{S}(M)$, then, using successively (2.13), (2.14), and (2.12), we get

$$
\begin{aligned}
& J_{X} \star_{\lambda} F-F \star_{\lambda} J_{X}=\left(\mathcal{Q}_{\lambda}\right)^{-1}\left[\mathcal{Q}_{\lambda}\left(J_{X}\right), \mathcal{Q}_{\lambda}(F)\right] \\
& =\left(\mathcal{Q}_{\lambda}\right)^{-1}\left[i \hbar L_{X}^{\lambda}, \mathcal{Q}_{\lambda}(F)\right] \\
& =i \hbar L_{X} F \\
& =i \hbar\left\{J_{X}, F\right\}
\end{aligned}
$$

where the last equality stems from the definition of the moment map. The proof that (2.3) holds is complete.

\section{Projectively/conformally invariant operators}

We gather here definitions and results that will be used throughout the paper. Those mainly concern projective/conformal differential geometry. We will consider the Lie groups $\mathfrak{G}=\mathrm{SL}(n+1, \mathbb{R})$ and $\mathfrak{G}=\mathrm{SO}_{0}(p+1, q+1)$ together with their homogeneous spaces $M=\mathbb{R} P^{n}$ and $M=\left(S^{p} \times S^{q}\right) / \mathbb{Z}_{2}$, respectively. From here on, $\mathfrak{G}$ will stand for either of the two groups above and $M$ for either of the corresponding homogeneous spaces. In the framework of Weyl's invariant theory [46], we will introduce, for each geometry, $\mathfrak{G}$-invariant linear operators on $T^{*} M$ which will serve as our main tools. 


\subsection{The projective and conformal symmetries}

The real projective space of dimension $n$ is an $\mathrm{SL}(n+1, \mathbb{R})$-homogeneous space. More precisely, $\mathbb{R P}^{n}=\mathrm{SL}(n+1, \mathbb{R}) / \operatorname{Aff}(n, \mathbb{R})$, where $\operatorname{Aff}(n, \mathbb{R})=\mathrm{GL}(n, \mathbb{R}) \ltimes \mathbb{R}^{n}$ is an affine subgroup of $\mathrm{SL}(n+1, \mathbb{R})$.

Let $x^{1}, x^{2}, \ldots, x^{n}$ be an affine coordinate system on $\mathbb{R P}^{n}$, the fundamental vector fields associated with the $\mathrm{SL}(n+1, \mathbb{R})$-action on $\mathbb{R} \mathrm{P}^{n}$ are then given by :

$$
\frac{\partial}{\partial x^{i}}, \quad x^{i} \frac{\partial}{\partial x^{j}}, \quad x^{i} x^{j} \frac{\partial}{\partial x^{j}},
$$

with $i, j=1, \ldots, n$. The vector fields (3.1) correspond to translations, linear transformations and inversions, respectively; they generate a flag of Lie algebras

$$
\mathbb{R}^{n} \subset \operatorname{aff}(n, \mathbb{R}) \subset \operatorname{sl}(n+1, \mathbb{R}) .
$$

The sphere $S^{n}$ with its canonical metric is a conformally flat manifold. The same is true for $\left(S^{p} \times S^{q}\right) / \mathbb{Z}_{2}$ in the case of signature $p-q$. Those are homogeneous spaces $\mathrm{SO}(p+1, q+1) / \mathrm{CE}(p, q)$ where $\mathrm{CE}(p, q)=\mathrm{CO}(p, q) \ltimes \mathbb{R}^{n}$ is the conformal Euclidean group, $\mathrm{CO}(p, q)=\mathrm{SO}(p, q) \rtimes \mathbb{R}_{+}^{*}$, and $n=p+q$.

The fundamental vector fields associated with the $\mathrm{SO}_{0}(p+1, q+1)$-action on $\left(S^{p} \times S^{q}\right) / \mathbb{Z}_{2}$ in an "anallagmatic" coordinate system are given (see, e.g., [19]) by

$$
\frac{\partial}{\partial x^{i}}, \quad x_{i} \frac{\partial}{\partial x^{j}}-x_{j} \frac{\partial}{\partial x^{i}}, \quad x^{i} \frac{\partial}{\partial x^{i}}, \quad x_{j} x^{j} \frac{\partial}{\partial x^{i}}-2 x_{i} x^{j} \frac{\partial}{\partial x^{j}}
$$

where $i, j=1, \ldots, n$ and where indices are raised and lowered using the standard metric $g$ of $\left(S^{p} \times S^{q}\right) / \mathbb{Z}_{2}$. The vector fields (3.2) correspond to translations, rotations, homotheties and inversions, respectively; they generate a flag of Lie algebras

$$
\mathbb{R}^{n} \subset \mathrm{e}(p, q) \subset \operatorname{ce}(p, q) \subset \mathrm{o}(p+1, q+1) .
$$

These two groups of transformations, $\mathfrak{G}$, define respectively the projective and the conformal geometries; their Lie algebras, $\mathfrak{g}$, spanned by the vector fields (3.1) and (3.2) are finite-dimensional maximal Lie subalgebras of $\operatorname{Vect}(M)$, see [37, 5].

We also introduce, for convenience, the notation $\mathfrak{H} \subset \mathfrak{G}$ for the affine Lie subgroups $\mathfrak{H}=\operatorname{Aff}(n, \mathbb{R})$ in the projective case, and $\mathfrak{H}=\mathrm{CE}_{0}(p, q)$ in the conformal case. The corresponding Lie subalgebras will be denoted by $\mathfrak{h} \subset \mathfrak{g}$. 


\subsection{Affine and Euclidean invariant operators}

Since the group Diff $(M)$ of diffeomorphisms of $M$ admits a canonical lift to $T^{*} M$, let us lift, accordingly, the action of $\mathfrak{G}$. The search for $\mathfrak{G}$-invariant linear operators on $\mathcal{S}(M)$ will be dealt with in two stages. We first consider the affine (resp. Euclidean) subgroup and determine the algebra of $\operatorname{Aff}(n, \mathbb{R})$-invariant $\left(\right.$ resp. $\left(\mathrm{SO}_{0}(p, q) \ltimes \mathbb{R}^{n}\right)$ invariant) operators; in the next section we will then enforce full $\mathfrak{G}$-invariance.

A classical result from invariant theory shows that the commutant of $\operatorname{Aff}(n, \mathbb{R})$ in $\operatorname{End}(\mathcal{S}(M))$ is generated by the following two operators

$$
\mathcal{E}=\xi_{i} \frac{\partial}{\partial \xi_{i}}, \quad \mathrm{D}=\frac{\partial}{\partial x^{i}} \frac{\partial}{\partial \xi_{i}}
$$

which span the Lie algebra aff $(1, \mathbb{R})$. Indeed, an $\operatorname{Aff}(n, \mathbb{R})$-invariant linear operator mapping $\mathcal{S}_{k}(M)$ into $\mathcal{S}_{\ell}(M)$ is proportional to $\mathrm{D}^{k-\ell}$ (see, e.g., [46, 29]). The commutant of $\operatorname{Aff}(n, \mathbb{R})$ in $\operatorname{End}(\mathcal{S}(M))$ is, hence, given by series in $\mathcal{E}$ and D, convergent on $\mathcal{S}(M)$.

It has been shown in 23] that the commutant of $\mathrm{SO}_{0}(p, q) \ltimes \mathbb{R}^{n}$ in $\operatorname{End}(\mathcal{S}(M))$ is generated by the operators

$$
\mathrm{R}=\xi^{i} \xi_{i}, \quad \mathcal{E}=\xi_{i} \frac{\partial}{\partial \xi_{i}}, \quad \mathrm{~T}=\frac{\partial}{\partial \xi^{i}} \frac{\partial}{\partial \xi_{i}}
$$

whose commutation relations are those of $\operatorname{sl}(2, \mathbb{R})$, together with

$$
\mathrm{G}=\xi^{i} \frac{\partial}{\partial x^{i}}, \quad \mathrm{D}=\frac{\partial}{\partial x^{i}} \frac{\partial}{\partial \xi_{i}}, \quad \Delta=\frac{\partial}{\partial x^{i}} \frac{\partial}{\partial x_{i}}
$$

which span the Heisenberg Lie algebra $h_{1}$. The operators (3.4) and (3.5) span the Lie algebra $\operatorname{sl}(2, \mathbb{R}) \ltimes \mathrm{h}_{1}$.

\subsection{Projectively and conformally invariant operators}

It is noteworthy that $\mathcal{E}$ commutes with the lift of any diffeomorphism of $M$. One may ask if, upon restriction to $\mathfrak{G} \subset \operatorname{Diff}(M)$, there exist other linear operators on $T^{*} M$ that commute with $\mathfrak{G}$. The answer is negative in the projective case and positive in the conformal case.

Proposition 3.1. The commutant of $\operatorname{SL}(n+1, \mathbb{R})$ in $\operatorname{End}(\mathcal{S}(M))$ is generated by $\mathcal{E}$. 
Proof. An affinely invariant linear operator is a series in $\mathcal{E}$ and $\mathrm{D}$ of the form

$$
A=\sum_{s=0}^{\infty} P_{s}(\mathcal{E}) \mathrm{D}^{s},
$$

where $P_{s}$ is a series in one variable. Let $X_{i}=x^{i} x^{j} \partial / \partial x^{j}$ be the $i$-th generator of inversions in (3.1). Straightforward computation (see [37]) yields the commutation relation

$$
\left[L_{X_{i}}, \mathrm{D}\right]=(2 \mathcal{E}+n+1) \circ \frac{\partial}{\partial \xi_{i}} .
$$

One then checks that

$$
\left[L_{X_{i}}, A\right]=\sum_{s=0}^{\infty} s P_{s}(\mathcal{E})(2 \mathcal{E}+n+s) \mathrm{D}^{s-1} \circ \frac{\partial}{\partial \xi_{i}} .
$$

This expression vanishes if and only if $P_{s}=0$ for all $s \geq 1$. Hence $A=P_{0}(\mathcal{E})$ is a necessary condition for $A$ to commute with the $\mathrm{SL}(n+1, \mathbb{R})$-action.

The conformal counterpart of the above statement is as follows.

Proposition 3.2. The commutant of $\mathrm{SO}_{0}(p+1, q+1)$ in $\operatorname{End}(\mathcal{S}(M))$ is the commutative associative algebra generated by $\mathcal{E}$ and the operator $\mathrm{R}_{0}=\mathrm{R} \circ \mathrm{T}$.

Proof. A sketch of this proof was given in [23]; for the sake of completeness we give here the details.

Let us consider an operator $Z$ on the space of polynomials of degree $k$, namely

$$
\mathcal{S}^{k}(M)=\bigoplus_{\ell=0}^{k} \mathcal{S}_{\ell}(M)
$$

and commuting with the canonical lift of $\mathrm{SO}_{0}(p+1, q+1)$. It is, according to classical invariant theory [46, 29], a differential operator, polynomial in the generators (3.4) and (3.5).

We therefore seek a differential operator $Z$ on $T^{*} M$ which commutes with the $\mathrm{SO}_{0}(p+1, q+1)$-action. Its principal symbol $\sigma(Z)$ is a function on $T^{*}\left(T^{*} M\right)$, polynomial on fibers. More precisely, if $\left(\zeta_{i}, y^{i}\right)$ denote the conjugate variables to $\left(x^{i}, \xi_{i}\right)$ respectively, then $\sigma(Z)$ is polynomial in the variables $\xi_{i}, \zeta_{i}, y^{i}$. The function $\sigma(Z)$ has to be annihilated by the canonical lifts to $T^{*}\left(T^{*} M\right)$ of all generators (3.2) of the conformal Lie algebra $\mathrm{o}(p+1, q+1)$. 
Let us assume that $\sigma(Z)$ is ce $(p, q)$-invariant and consider then invariance with respect to inversions whose $i$-th generator is $X_{i}=x_{j} x^{j} \partial / \partial x^{i}-2 x_{i} x^{j} \partial / \partial x^{j}$. Its canonical lift to $T^{*}\left(T^{*} M\right)$ is given by

$$
\begin{aligned}
\widetilde{L}_{X_{i}}= & x_{j} x^{j} \frac{\partial}{\partial x^{i}}-2 x_{i} x^{j} \frac{\partial}{\partial x^{j}} \\
& +2 x_{i}\left(\xi_{j} \frac{\partial}{\partial \xi_{j}}-y^{j} \frac{\partial}{\partial y^{j}}+\zeta_{j} \frac{\partial}{\partial \zeta_{j}}\right) \\
& -2 x^{j}\left(\xi_{i} \frac{\partial}{\partial \xi^{j}}-\xi_{j} \frac{\partial}{\partial \xi^{i}}+y_{i} \frac{\partial}{\partial y^{j}}-y_{j} \frac{\partial}{\partial y_{i}}+\zeta_{i} \frac{\partial}{\partial \zeta^{j}}-\zeta_{j} \frac{\partial}{\partial \zeta^{i}}\right) \\
& +2\left(\xi_{i} y_{j} \frac{\partial}{\partial \zeta_{j}}-y_{i} \xi_{j} \frac{\partial}{\partial \zeta_{j}}-\xi_{j} y^{j} \frac{\partial}{\partial \zeta^{i}}\right)
\end{aligned}
$$

and the invariance with respect to inversions reads $\widetilde{L}_{X_{i}} \sigma(Z)=0$. Now, invariance with respect to $\operatorname{ce}(p, q)$ clearly implies that $\sigma(Z)$ is annihilated by the first three terms in (3.8), so that

$$
\left(\xi_{i} y_{j} \frac{\partial}{\partial \zeta_{j}}-y_{i} \xi_{j} \frac{\partial}{\partial \zeta_{j}}-\xi_{j} y^{j} \frac{\partial}{\partial \zeta^{i}}\right) \sigma(Z)=0
$$

for all $i=1, \ldots, n$.

Lemma 3.3. The equation (3.9) implies

$$
\frac{\partial \sigma(Z)}{\partial \zeta_{i}}=0
$$

for all $i=1, \ldots, n$.

Proof. The determinant of the matrix

$$
A_{j}^{i}=y^{i} \xi_{j}-\xi^{i} y_{j}+\xi_{k} y^{k} \delta_{j}^{i}
$$

intervening in (3.9) is $\operatorname{det}(A)=\xi_{i} \xi^{i} y_{j} y^{j}\left(\xi_{k} y^{k}\right)^{n-2}$ which is non-zero on the complement of a lower-dimensional smooth submanifold of $T^{*}\left(T^{*} M\right)$.

By e $(p, q)$-invariance, the operator $Z$ is a polynomial in the differential operators (3.4) and (3.5), see Section 3.2. Furthermore, invariance with respect to the generator of homotheties $X_{0}=x^{i} \partial / \partial x^{i}$ shows that $Z$ is in fact a polynomial in

$$
\mathrm{R}_{0}=\mathrm{R} \circ \mathrm{T}, \quad \mathcal{E}, \quad \mathrm{G}_{0}=\mathrm{G} \circ \mathrm{T}, \quad \mathrm{D}, \quad \Delta_{0}=\Delta \circ \mathrm{T}
$$


The principal symbols of the last three operators are

$$
\sigma\left(\mathrm{G}_{0}\right)=\xi_{i} \zeta^{i} y_{j} y^{j}, \quad \sigma(\mathrm{D})=\zeta_{i} y^{i}, \quad \sigma\left(\Delta_{0}\right)=\zeta_{i} \zeta^{i} y_{j} y^{j} .
$$

These three polynomials are algebraically independent for $n>1$. Condition (3.10) implies then that $Z$ depends only on $\mathcal{E}$ and $\mathrm{R}_{0}$. Note that if $n=1$, we find $\mathrm{R}_{0}=$ $\mathcal{E}(\mathcal{E}-1)$ in agreement with Proposition 3.1 .

We have thus shown that, for all $k$, any $Z \in \operatorname{End}\left(\mathcal{S}^{k}(M)\right)$ commuting with the $\mathrm{SO}_{0}(p+1, q+1)$-action is polynomial in $\mathcal{E}$ and $\mathrm{R}_{0}$. This completes the proof of Proposition 3.2

\section{Existence of projectively and conformally in- variant star-products}

Taking advantage of the results obtained in equivariant quantization (see [37, 23, 9]) and of Proposition 2.9. one defines a $\mathfrak{G}$-invariant star-product on $T^{*} M$. In this section we give a brief account on the projectively and conformally equivariant quantizations and discuss the main properties of the associated invariant star-products.

\subsection{Construction of $\mathfrak{G}$-invariant star-products}

It has been proved in 37, 23. that, for any $\lambda \in \mathbb{C}$, there exists a unique $\mathfrak{G}$-equivariant quantization map $\mathcal{Q}_{\lambda}: \mathcal{S}(M) \rightarrow \mathcal{D}_{\lambda}(M)[\hbar]$ on $T^{*} M$.

In a local coordinate system, one can locally identify $\mathcal{S}(M)$ and $\mathcal{D}_{\lambda}(M)$ through the normal ordering prescription:

$$
P^{i_{1} \ldots i_{k}} \xi_{i_{1}} \cdots \xi_{i_{k}} \mapsto(i \hbar)^{k} P^{i_{1} \ldots i_{k}} \frac{\partial}{\partial x^{i_{1}}} \cdots \frac{\partial}{\partial x^{i_{k}}}
$$

where $P^{i_{1} \ldots i_{k}}$ is a smooth function of $\left(x^{1}, \ldots, x^{n}\right)$.

The explicit formula of $\mathcal{Q}_{\lambda}$ is only known in the projective case; it is given, in an adapted coordinate system, and using the identification (4.1), by the series 22

$$
\mathcal{Q}_{\lambda}=\sum_{r=0}^{\infty} C_{r}(\mathcal{E})(i \hbar \mathrm{D})^{r}
$$

where $\mathcal{E}$ and $\mathrm{D}$ are as in $(3.3)$ and

$$
C_{r}(\mathcal{E})=\frac{1}{r !} \frac{(\mathcal{E}+(n+1) \lambda)_{r}}{(2 \mathcal{E}+n+r)_{r}}
$$


where $(a)_{r}:=a(a+1) \cdots(a+r-1)$ is the Pochhammer symbol. The expression (4.2) is well defined globally on $T^{*} M$ since $M$ is projectively flat.

An important feature of the quantization map (4.2) is that it is homogeneous in the following sense. Let us assign a degree to the deformation parameter $\hbar$, more precisely, we put

$$
\operatorname{deg} \hbar=1
$$

Then $\mathcal{Q}_{\lambda}$ preserves the total degree on $\mathcal{S}(M)[\hbar]$. In other words, one has

Proposition 4.1. The quantization map $\mathcal{Q}_{\lambda}$ commutes with the Euler operator:

$$
\widehat{\mathcal{E}}=\mathcal{E}+\hbar \frac{\partial}{\partial \hbar}
$$

Proof. This follows from the commutation relation $[\mathcal{E}, \mathrm{D}]=-\mathrm{D}$ and the expression (4.2).

In the conformal case we have no explicit formula for the $\mathrm{SO}_{0}(p+1, q+1)$ equivariant quantization map. However, one can guarantee [23] that $\mathcal{Q}_{\lambda}$ is also homogeneous in this case.

A $\mathfrak{G}$-invariant star-product on $T^{*} M$ can be obtained from such a $\mathfrak{G}$-equivariant quantization map.

Proposition 4.2 ([23, 9]). The associative product associated with $\mathcal{Q}_{\lambda}$ through (2.13) is a star-product if and only if $\lambda=\frac{1}{2}$.

The proof consists in checking that $\lambda=\frac{1}{2}$ is the only value of $\lambda$ for which the first-order term in $\hbar$ of the associative product (2.13) coincides with the Poisson bracket.

Note, however, that the uniqueness of $\mathcal{Q}_{\frac{1}{2}}$ does not a priori insure the uniqueness of a $\mathfrak{G}$-invariant star-product.

\subsection{Main properties}

For the constructed $\mathfrak{G}$-invariant star-products Condition $\mathrm{C} 1$ from Section 1 is satisfied. We will show below that Condition $\mathrm{C} 2$ also holds. 
Definition 4.3. A star-product on the space $\mathcal{S}(M)$ will be called homogeneous, if all the bilinear operators $B_{r}$ in (1.1) are homogeneous of degree $r$, that is, if they preserve the grading (2.7) according to

$$
B_{r}: \mathcal{S}_{k}(M) \otimes \mathcal{S}_{\ell}(M) \rightarrow \mathcal{S}_{k+\ell-r}(M)
$$

or, equivalently, if $\widehat{\mathcal{E}}$ is a derivation of the star-product algebra.

Proposition 4.4. The $\mathfrak{G}$-invariant star-product (2.13) obtained from the $\mathfrak{G}$-equivariant quantization map $\mathcal{Q}_{\frac{1}{2}}$ is symmetric, homogeneous and strongly $\mathfrak{G}$-invariant.

Proof. The quantization map $\mathcal{Q}_{\frac{1}{2}}$ is symmetric, namely, it satisfies

$$
\mathcal{Q}_{\frac{1}{2}}(F)^{*}=\mathcal{Q}_{\frac{1}{2}}(\bar{F})
$$

for all $F \in \mathcal{S}(M)$ [37, 23, 21], where $\mathcal{Q}_{\frac{1}{2}}(F)^{*}$ denotes the formal adjoint operator with respect to the natural pairing on compactly supported $\frac{1}{2}$-densities. Using the definition (2.13) of the star-product, one now gets the symmetry condition $\mathrm{C} 2$.

Homogeneity of the quantization map $\mathcal{Q}_{\frac{1}{2}}$ readily implies the homogeneity of the corresponding star-product.

The projectively and the conformally equivariant quantization maps $\mathcal{Q}_{\frac{1}{2}}$ coincide up to second-order terms, namely, in both cases one has

$$
\mathcal{Q}_{\frac{1}{2}}=\mathrm{Id}+\frac{i \hbar}{2} \mathrm{D}+O\left(\hbar^{2}\right)
$$

in any coordinate system (cf. [37, 23]). One easily verifies that $\mathcal{Q}_{\frac{1}{2}}$ satisfies condition (2.14). By Proposition 2.10, the associated $\mathfrak{G}$-invariant star-products are thus strongly $\mathfrak{G}$-invariant.

Condition C3 fails to be satisfied (as proved in [9] and [2] for a subalgebra of $\mathcal{S}(M))$. Each term $B_{r}$ is a pseudo-differential bilinear operator, while its restriction $\left.B_{r}\right|_{\mathcal{S}_{k}(M) \otimes \mathcal{S}_{\ell}(M)}$ is a bidifferential operator, just like $\left.\mathcal{Q}_{\frac{1}{2}}\right|_{\mathcal{S}_{k}(M)}$ is a differential operator, see [37]. Hence the constructed star-product is local, namely, for all $F, G \in \mathcal{S}(M), \operatorname{Supp}(F \star G) \subset \operatorname{Supp}(F) \cap \operatorname{Supp}(G)$, see Lemma 5.3 below. 


\section{Uniqueness of $\mathfrak{G}$-invariant star-product}

Our goal is to show that the star-products constructed in Section 4.1] with the help of the $\mathfrak{G}$-equivariant quantization map are the unique $\mathfrak{G}$-invariant star-products where, as above, $\mathfrak{G}=\mathrm{SL}(n+1, \mathbb{R})$ and $\mathfrak{G}=\mathrm{SO}_{0}(p+1, q+1)$, respectively. We prove uniqueness in two different settings:

1. in the class of homogeneous $\mathfrak{G}$-invariant star-products,

2. in the class of all $\mathfrak{G}$-invariant star-products modulo formal reparametrizations and $\mathfrak{G}$-equivalence.

\subsection{Homogeneous star-products}

Homogeneity of a star-product (see Definition 4.3) is a very natural property from a physical standpoint. Indeed, if one considers $\hbar$ as a physical constant whose dimension is that of an action (i.e., the dimension of Planck's constant which is also the inverse dimension of the Poisson bracket on $\left.T^{*} M\right)$ then the physical dimension of the star-product $F \star G$ of two observables is the same as that of their product $F G$, when $\star$ is homogeneous. This is a direct consequence of the fact that $B_{r}$ has the same physical dimension as $\hbar^{-r}$, which follows from associativity. In other words a homogeneous star-product is dimensionless.

On the other hand, homogeneous star-products were thoroughly studied in the mathematical literature. For instance, De Wilde and Lecomte proved [15] that any two homogeneous star-products on a cotangent bundle are equivalent (in the sense of the definitions of Section 2.21). The $\mathfrak{G}$-invariant star-products constructed in Section 4.1 are also homogeneous (see Proposition 4.4).

The first main result of this paper is

Theorem 5.1. There exists a unique homogeneous $\mathfrak{G}$-invariant star-product on the space of symbols $\mathcal{S}(M)$.

Proof. In Section 4 we proved the existence of a homogeneous $\mathfrak{G}$-invariant starproduct on $\mathcal{S}(M)$. We will now prove its uniqueness.

Let $\star$ and $\star^{\prime}$ be two homogeneous $\mathfrak{G}$-invariant star-products. Let us assume that the first $r-1$ terms of these star-products coincide, and use induction over $r$. 
The difference $B_{r}-B_{r}^{\prime}$ is a $\mathfrak{G}$-invariant homogeneous Hochschild 2-cocycle. Indeed, associativity of the star-product $\star$ implies that $\delta B_{r}$ depends only upon $B_{i}$ with $i<r$, where the Hochschild coboundary of a 2-cochain $B$ is given by

$$
\delta B(F, G, H)=F B(G, H)-B(F G, H)+B(F, G H)-B(F, G) H,
$$

implying that $\delta\left(B_{r}-B_{r}^{\prime}\right)=0$.

Let $C$ be a Hochschild 2-cocycle on $\mathcal{S}(M)$. Assume now that $C$ is homogeneous as in (4.5) and $\mathfrak{G}$-invariant. As a bilinear map, $C$ decomposes as a sum $C_{1}+C_{0}$, where $C_{1}$ and $C_{0}$ are, respectively, the skew-symmetric and the symmetric parts of $C$. We will need the following well-known result.

Proposition 5.2. For any local Hochschild 2-cocycle $C$ on $\mathcal{S}(M)$, the skew-symmetric part $C_{1}$ is a bivector, and the symmetric part $C_{0}$ is the coboundary of a local 1-cochain.

This statement is an important result in deformation theory. It was first established in the differentiable case [44] and was later on generalized to local cocycles in [10] (let us mention that this result also holds for continuous cocycles [13, 40]).

In order to apply Proposition 5.2, we will prove that each term $B_{r}$ of a $\mathfrak{G}$ invariant star-product is local, a result that generalizes Theorem 5.1 in [37.

Lemma 5.3. Any linear $\mathfrak{G}$-invariant operator $B: \mathcal{S}_{k}(M) \otimes \mathcal{S}_{\ell}(M) \rightarrow \mathcal{S}_{m}(M)$ with $m \leq k+\ell$ is local.

Proof. We must prove that $\operatorname{Supp}(B(F, G)) \subset \operatorname{Supp}(F) \cap \operatorname{Supp}(G)$ for all $F \in \mathcal{S}_{k}(M)$ and $G \in \mathcal{S}_{\ell}(M)$. Suppose that one of the arguments, $F$ or $G$, vanishes in a neighbourhood of some $x \in M$; we will prove that $B(F, G)(x)=0$. Let us now locally identify $M$ with $\mathbb{R}^{n}$ and consider the subalgebra $\mathbb{R} \ltimes \mathbb{R}^{n}$ of $\mathfrak{g}$ generated by the Euler vector field, $\mathcal{E}$, and the translations. Using translation-invariance, we may, hence, assume $x=0$.

We will embed $\mathcal{S}_{k}\left(\mathbb{R}^{n}\right) \otimes \mathcal{S}_{\ell}\left(\mathbb{R}^{n}\right)$ into $\mathcal{S}_{k+\ell}\left(\mathbb{R}^{2 n}\right)$ and notice that $F \otimes G$ vanishes in a neighbourhood of $x=0$ in $\mathbb{R}^{2 n}$. It remains to show that if $B: \mathcal{S}_{k+\ell}\left(\mathbb{R}^{2 n}\right) \rightarrow \mathcal{S}_{m}\left(\mathbb{R}^{n}\right)$ is a linear map which commutes with the action of homotheties $L_{\mathcal{E}}$, then for all $H \in \mathcal{S}_{k+\ell}\left(\mathbb{R}^{2 n}\right)$ that vanishes in a neighbourhood of $x=0$, we have $B(H)(0)=0$ provided $m \leq k+\ell$. But the proof of the latter statement coincides with that of Theorem 5.1 in [37]. 
The building blocks of the operators $B_{r}$ are the $\mathfrak{H}$-invariant operators listed in (3.11). These operators never increase the degree of homogeneity in $\xi=\left(\xi_{i}\right)$, hence Lemma 5.3 applies. We are now able to use Proposition 5.2 and consider $C_{1}$ and $C_{0}$ separately. The assertion of Theorem 5.1] will follow from Lemmas 5.4 and 5.6 below.

Lemma 5.4. There is no non-zero $\mathfrak{G}$-invariant bivector on $T^{*} M$ with coefficients in $\mathcal{S}(M)$ homogeneous of degree $r \geq 2$.

Proof. There is clearly no non-zero such bivector $W: \mathcal{S}_{k}(M) \otimes \mathcal{S}_{\ell}(M) \rightarrow \mathcal{S}_{k+\ell-r}(M)$, for $r>2$. For $r=2$, if it exists it is necessarily of the form $W=W_{i j} \partial / \partial \xi_{i} \wedge \partial / \partial \xi_{j}$ with coefficients $W_{i j}$ of degree 0 in $\xi$. Since $W$ is invariant with respect to the generators of translations, $\partial W_{i j} / \partial x^{s}=0$ for all $s=1, \ldots, n$. But, in this case, $W$ cannot be invariant with respect to homotheties.

We thus have proved that there is no non-zero bivector invariant with respect to the $(n+1)$-dimensional Lie algebra of translations and homotheties. This Lie algebra is a Lie subalgebra of both $\operatorname{sl}(n+1, \mathbb{R})$ and $o(p+1, q+1)$. Lemma 5.4 is proved.

Remark 5.5. Note that, in the proofs of Lemmas [5.3] and [5.4, we only needed invariance with respect to a subalgebra of $\mathfrak{g}$.

Lemma 5.6. There is no non-zero $\mathfrak{G}$-invariant Hochschild 2-coboundary $C_{0}$ on the associative commutative algebra $\mathcal{S}(M)$ homogeneous of degree $r \geq 2$.

Proof. Suppose that such a $C_{0}$ exists. Being a coboundary, it is of the form $C_{0}=\delta A$ where

$$
\delta A(F, G)=F A(G)-A(F G)+A(F) G
$$

for some linear map $A: \mathcal{S}_{k}(M) \rightarrow \mathcal{S}_{k-r}(M)$, with $r \geq 2$. Let us prove that $A$ is $\mathfrak{G}$-invariant.

Since $C_{0}$ is $\mathfrak{G}$-invariant, then, for any $X \in \mathfrak{g}$, the linear map $L_{X}(A)=\left[L_{X}, A\right]$ is a Hochschild 1-cocycle on $\mathcal{S}(M)$. Indeed one has $\delta \circ L_{X}=L_{X} \circ \delta$. Thus, $L_{X}(A)$ is a derivation on $\mathcal{S}(M)$. Therefore, this is a vector field on $T^{*} M$ polynomial in $\xi$ and, hence, $L_{X}(A)$ cannot decrease the degree by more than 1 . Note, however, that $L_{X}(A): \mathcal{S}_{k}(M) \rightarrow \mathcal{S}_{k-r}(M)$ with $r \geq 2$ since, again, $L_{X}(A)=L_{X} \circ A-A \circ L_{X}$ 
and $L_{X}$ preserves $\mathcal{S}_{k}(M)$ for any vector field $X$ on $M$. It follows that $L_{X}(A)=0$ for all $X \in \mathfrak{g}$ and thus $A$ is $\mathfrak{G}$-invariant.

The classification of $\mathfrak{G}$-invariant linear maps on $\mathcal{S}(M)$ is given by Proposition 3.1 and Proposition 3.2. Being homogeneous of degree zero in $\xi$, a non-zero $\mathfrak{G}$-invariant element $A$ of $\operatorname{End}(\mathcal{S}(M))$ cannot decrease the degree. Lemma 5.6] is proved.

Lemmas 5.4 and 5.6 imply that $B_{r}-B_{r}^{\prime}=0$ for $r \geq 2$. This completes the proof of Theorem 5.1 .

The unique homogeneous $\mathfrak{G}$-invariant star-product will be called $\mathfrak{G}$-canonical. According to Proposition 4.4 this $\mathfrak{G}$-canonical star-product is the one associated with the $\mathfrak{G}$-equivariant quantization map $\mathcal{Q}_{\frac{1}{2}}$ from Section 4 . The same Proposition also states that it is both symmetric and strongly $\mathfrak{G}$-invariant.

\subsection{Uniqueness up to $\mathfrak{G}$-equivalence and reparametrization}

The following theorem is the second main result of this paper.

Theorem 5.7. The $\mathfrak{G}$-canonical star-product on the space of symbols $\mathcal{S}(M)$ is the unique $\mathfrak{G}$-invariant star-product modulo formal reparametrizations and $\mathfrak{G}$-equivalence.

Proof. Let $\star$ and $\star^{\prime}$ be two $\mathfrak{G}$-invariant star-products. Let us assume that there exists a $\mathfrak{G}$-invariant formal series (2.4) and a reparametrization (2.6) intertwining the first $r-1$ terms of these star-products, and use induction over $r$. Using this equivalence we can assume that $\star$ and $\star^{\prime}$ coincide up to the $(r-1)$-th order term. The difference $B_{r}-B_{r}^{\prime}$ is then a $\mathfrak{G}$-invariant Hochschild 2-cocycle.

As in Section 5.1 we consider the decomposition $C=C_{1}+C_{0}$, where $C_{1}$ and $C_{0}$ are, respectively, the skew-symmetric and the symmetric parts of $C$. By Proposition 5.2, $C_{1}$ is a bivector and $C_{0}$ is a coboundary.

We will need the following two lemmas.

Lemma 5.8. (i) In the projective case, the canonical Poisson bivector

$$
\Pi=\frac{\partial}{\partial \xi_{i}} \wedge \frac{\partial}{\partial x^{i}}
$$

on $T^{*} M$ is the unique (up to an overall multiplicative constant) $\mathfrak{G}$-invariant bivector. 
(ii) In the conformal case with $n \neq 2$, the canonical Poisson bivector on $T^{*} M$ is the unique (up to an overall multiplicative constant) $\mathfrak{G}$-invariant bivector.

(iii) In the conformal case with $n=2$, there are two $\mathfrak{G}$-invariant bivectors on $T^{*} M$, namely the canonical Poisson bivector and the Poisson bivector

$$
\Lambda=\frac{1}{2} \mathrm{~g}^{i j} \xi_{i} \xi_{j} \sigma_{k \ell} \frac{\partial}{\partial \xi_{k}} \wedge \frac{\partial}{\partial \xi_{\ell}}
$$

where $\mathrm{g}=\mathrm{g}_{i j} d x^{i} d x^{j}$ represents a conformal class of (pseudo-)Riemannian metrics and $\sigma=\frac{1}{2} \sigma_{k \ell} d x^{k} \wedge d x^{\ell}$ stands for the surface element of $(M, \mathrm{~g})$.

Proof. Consider an arbitrary bivector field $W$ on $T^{*} M$. In any local coordinate system it is of the form

$$
W=W^{i j}(\xi, x) \frac{\partial}{\partial x^{i}} \wedge \frac{\partial}{\partial x^{j}}+W_{i}^{j}(\xi, x) \frac{\partial}{\partial \xi_{i}} \wedge \frac{\partial}{\partial x^{j}}+W_{i j}(\xi, x) \frac{\partial}{\partial \xi_{i}} \wedge \frac{\partial}{\partial \xi_{j}}
$$

where the coefficients $W^{i j}(\xi, x), W_{i}^{j}(\xi, x)$ and $W_{i j}(\xi, x)$ are functions of $x^{i}, \xi_{i}$ which are polynomial in $\xi$.

Choose an adapted coordinate system related to the projective or conformal structure on $M$ respectively (see Section 3.1). Since $W$ is $\mathfrak{G}$-invariant, it commutes with the action of the generators of translations, that is, with the vector fields $X_{i}=\partial / \partial x^{i}$, where $i=1, \ldots, n$. It follows that the coefficients of $W$ are independent of $x^{i}$. Furthermore, $W$ is invariant with respect to the action of the homothety vector field $X_{0}=x^{i} \partial / \partial x^{i}$. The canonical lift of $X_{0}$ to $T^{*} M$ is $L_{X_{0}}=x^{i} \partial / \partial x^{i}-\xi_{i} \partial / \partial \xi_{i}$. One immediately obtains the following homogeneity conditions:

1. the coefficient $W^{i j}(\xi)$ has to be homogeneous in $\xi$ of degree -2 ,

2. the coefficient $W_{i}^{j}(\xi)$ has to be homogeneous in $\xi$ of degree 0 ,

3. the coefficient $W_{i j}(\xi)$ has to be homogeneous in $\xi$ of degree 2 , so that $W^{i j}(\xi)=0$, while $W_{i}^{j}(\xi)$ are constant, and $W_{i j}(\xi)=W_{i j}^{k \ell} \xi_{k} \xi_{\ell}$ are quadratic polynomials. A $\mathfrak{G}$-invariant bivector (5.5) is, therefore, a sum of two independent $\mathfrak{G}$-invariant bivectors $W_{0}=W_{i}^{j} \partial / \partial \xi_{i} \wedge \partial / \partial x^{j}$ and $W_{2}=W_{i j}^{k \ell} \xi_{k} \xi_{\ell} \partial / \partial \xi_{i} \wedge \partial / \partial \xi_{j}$.

Considering now invariance with respect to the linear subgroup of $\mathfrak{G}$ entails that $W_{0}$ represents an invariant in $\left(\mathbb{R}^{n}\right)^{*} \otimes \mathbb{R}^{n}$ and $W_{2}$ an invariant in $\Lambda^{2}\left(\mathbb{R}^{n}\right)^{*} \otimes S^{2} \mathbb{R}^{n}$ with respect to the standard linear action of $\operatorname{SL}(n, \mathbb{R})$ in the projective case, and $\operatorname{SO}_{0}(p, q)$ 
in the conformal case. A classical result of invariant theory (see [46, 29]) yields $W_{0}=c_{0} \Pi$ with $c_{0} \in \mathbb{C}$ and $W_{2}=0$, except for $n=2$, in the conformal case, where $W_{2}=c_{2} \Lambda$ with $c_{2} \in \mathbb{C}$. Hence, we have proved that the bivectors (5.3), and (5.4) for $n=2$ in the conformal case, are the only bivectors invariant with respect to the affine subgroup of $\mathfrak{G}$.

To complete the proof, one checks that the bivectors (15.3) and (5.4) are invariant with respect to inversions, i.e., the quadratic vector fields in (3.1) and (3.2).

Lemma 5.9. Every $\mathfrak{G}$-invariant Hochschild 2-coboundary $C_{0}$ on the associative commutative algebra $\mathcal{S}(M)$ is of the form $C_{0}=\delta A$ where $A$ is a $\mathfrak{G}$-invariant linear map on $\mathcal{S}(M)$.

Proof. The 2-coboundary $C_{0}$ is local thanks to Lemma 5.3. This clearly implies that any 1-cochain $A$ such that $C_{0}=\delta A$ is local, cf. Proposition 5.2.

Given a $\mathfrak{G}$-invariant Hochschild 2-coboundary $C_{0}=\delta A$, we will prove that there exists a linear map $\widetilde{A}$ such that $\delta \widetilde{A}=\delta A$ and $L_{X}(\widetilde{A})=0$ for all $X \in \mathfrak{g}$. Clearly, $\mathfrak{G}$-invariance of $C_{0}=\delta A$ implies $L_{X}(\delta A)=0$ for any $X \in \mathfrak{g}$. Thus, $\delta\left(L_{X}(A)\right)=0$ which means that $L_{X}(A)$ is a vector field.

A local operator $A$ is a locally given, according to the Peetre theorem [42], by a differential operator; in an arbitrary coordinate system,

$$
A=A^{(0)}+A^{(1)}+A^{(2)}+\cdots+A^{(m)}
$$

where

$$
A^{(i)}=\sum_{i_{1}+i_{2}=i} A_{t_{1} \cdots t_{i_{2}}}^{s_{1} \ldots s_{i_{1}}}(x, \xi) \frac{\partial}{\partial x^{s_{1}}} \cdots \frac{\partial}{\partial x^{s_{1}}} \frac{\partial}{\partial \xi_{t_{1}}} \cdots \frac{\partial}{\partial \xi_{t_{i_{2}}}} .
$$

Choose a coordinate system adapted to either the projective or the conformal structure. Consider first the action of the affine Lie subalgebra, $\mathfrak{h} \subset \mathfrak{g}$, that is, $\mathfrak{h}=\operatorname{aff}(n, \mathbb{R})$ in the projective case and $\mathfrak{h}=\operatorname{ce}(p, q)$ in the conformal case, introduced in Section 3.1 .

For each component $A^{(i)}$, except for $A^{(1)}$, one has $L_{X}\left(A^{(i)}\right)=0$, where $X \in \mathfrak{h}$, since this operator is of the form (5.7) and thus cannot be a vector field. Put $\widetilde{A}=A-A^{(1)}$; this operator satisfies $L_{X}(\widetilde{A})=0$ for all $X \in \mathfrak{h}$ and, obviously, $\delta \widetilde{A}=\delta A$. In particular, invariance with respect to translations guarantees that the coefficients in (5.7) are independent of $x$. 
In the projective case, an affinely invariant operator $\widetilde{A}$ is of the form (3.6); for the generators $X_{i}$ of inversions, $L_{X_{i}}(\widetilde{A})$ is given by (3.7). This is a vector field if and only if $P_{s}=0$ for all $s \geq 1$. Hence $\widetilde{A}=P_{0}(\mathcal{E})$ and thus $L_{X_{i}}(\widetilde{A})=0$, see Proposition 3.1 .

In the conformal case, let us rewrite the expression of $\widetilde{A}$ in a different form, namely

$$
\widetilde{A}=\widetilde{A}_{(0)}+\widetilde{A}_{(1)}+\widetilde{A}_{(2)}+\cdots+\widetilde{A}_{(t)}
$$

where $t \leq m$ and

$$
\widetilde{A}_{(j)}=\widetilde{A}^{s_{1} \ldots s_{j}} \frac{\partial}{\partial x^{s_{1}}} \cdots \frac{\partial}{\partial x^{s_{j}}}
$$

each $\widetilde{A}^{s_{1} \ldots s_{j}}$ is a differential operator in $\xi$ with polynomial coefficients in $\xi$. Each term $\widetilde{A}_{(j)}$ is invariant with respect to translations and homogeneous in $x$ of degree $-j$. Invariance with respect to homotheties implies that $\widetilde{A}_{(j)}$ is homogeneous in $\xi$ of degree $-j$, that is,

$$
\left[\mathcal{E}, \widetilde{A}_{(j)}\right]=-j \widetilde{A}_{(j)}
$$

Let $X_{i}$ be the $i$-th generator of inversions. The operator $L_{X_{i}}\left(\widetilde{A}_{(j)}\right)$ is homogeneous in $\xi$ of degree $-j$, since $L_{X_{i}}(\mathcal{E})=0$, cf. Proposition 3.2. Hence, $L_{X_{i}}(\widetilde{A})$ is a vector field only if $L_{X_{i}}\left(\widetilde{A}_{(j)}\right)=0$ for $j \geq 2$ since it is polynomial in $\xi$.

Because of its $\mathfrak{h}$-invariance, $\widetilde{A}$ belongs to the ring generated by the operators

$$
\mathcal{E}, \quad \mathrm{R}_{0}=\mathrm{R} \circ \mathrm{T}, \quad \mathrm{D}, \quad \mathrm{G}_{0}=\mathrm{G} \circ \mathrm{T}, \quad \Delta_{0}=\Delta \circ \mathrm{T}
$$

where these operators have been defined in (3.4), (3.5) and (3.11). The term $\widetilde{A}_{(1)}$ is then necessarily of the form

$$
\widetilde{A}_{(1)}=\alpha \mathrm{D}+\beta \mathrm{G}_{0}
$$

where $\alpha$ and $\beta$ are polynomials in $\mathcal{E}$ and $\mathrm{R}_{0}$. A direct computation yields

$$
L_{X_{i}}\left(\widetilde{A}_{(1)}\right)=2 \alpha\left(\xi_{i} \mathrm{~T}-2 \mathcal{E} \frac{\partial}{\partial \xi_{i}}-n \frac{\partial}{\partial \xi_{i}}\right)-2 \beta\left(\mathrm{R}_{0} \frac{\partial}{\partial \xi_{i}}+2 \xi_{i} \mathrm{~T}\right)
$$

Every term in this expression, except for $-2 n \alpha \partial / \partial \xi_{i}$, is a differential operator of order $>1$ for any $\alpha$ and $\beta$. Thus, the right hand side can be a non-zero vector field only if $\alpha$ is a non-zero constant. On the other hand, $-2 \beta \mathrm{R}_{0} \partial / \partial \xi_{i}$ is, at least, a thirdorder term unless $\beta$ is zero. But, the remaining terms $2 \alpha \xi_{i} \mathrm{~T}$ and $-4 \alpha \mathcal{E} \partial / \partial \xi_{i}$ are of order 2 and linearly independent. One concludes that $\alpha=0$ and thus $L_{X_{i}}\left(\widetilde{A}_{(1)}\right)=0$. 
Finally, the term $\widetilde{A}_{(0)}$ is obviously a polynomial in $\mathcal{E}$ and $\mathrm{R}_{0}$ and, hence, $L_{X_{i}}\left(\widetilde{A}_{(0)}\right)=0$.

We have thus proved that $L_{X_{i}}(\widetilde{A})=0$ for all $i=1, \ldots, n$. Lemma [5.9] is proved.

Let us resort to Lemmas 5.8 and 5.9 to complete the proof. The $\mathfrak{G}$-invariant Hochschild 2-cocycle $C=B_{r}-B_{r}^{\prime}$ is a sum $C=C_{1}+C_{0}$.

The symmetric part $C_{0}$ is a Hochschild coboundary and, by Lemma 5.9] is of the form $C_{0}=\delta A$ where $A$ is a $\mathfrak{G}$-invariant 1-cochain. This term can be removed by a $\mathfrak{G}$-equivalence map $\Phi=\operatorname{Id}+(i \hbar)^{r} A$.

Under the hypotheses of parts (i) and (ii) of Lemma 5.8, the skew-symmetric part $C_{1}$ is proportional to the canonical Poisson bivector, that is, to the first-order term $B_{1}$. It can be removed by a reparametrization $i \hbar \mapsto i \hbar+c(i \hbar)^{r}$ for some $c \in \mathbb{R}$.

Theorem 5.7 is proved for the first two options, (i) and (ii), of Lemma 5.8,

In the conformal case and for $n=2$ (part (iii) of Lemma 5.8), the skewsymmetric part $C_{1}$ is a linear combination of the canonical Poisson bivector $\Pi$ and of the bivector $\Lambda$ in (5.4). By a reparametrization map we can remove the canonical Poisson bivector but not the bivector $\Lambda$.

Let us, indeed, show that, if $B_{r}-B_{r}^{\prime}=C_{1}=k \Lambda$, then necessarily $k=0$. We associate to the star-products $\star$ and $\star^{\prime}$ the corresponding star-commutators

$$
[F, G]_{\star}=\frac{1}{i \hbar}(F \star G-G \star F) .
$$

Since the two star-products are associative, the corresponding star-commutators satisfy the Jacobi identity. Put $J_{\star}(F, G, H)=\left[F,[G, H]_{\star}\right]_{\star}+($ cyclic) and consider the difference $J_{\star}(F, G, H)-J_{\star^{\prime}}(F, G, H)$. By assumption, this expression has to be identically zero. Since the two star-products coincide up to order $r-1$ in $i \hbar$, this difference is trivially zero up to order $r-2$. Straightforward computation shows that the $(r-1)$-th order term in the above difference is equal to $2 k[\Pi, \Lambda](F, G, H)$, where $[\Pi, \Lambda]$ is the Schouten bracket of $\Pi$ and $\Lambda$. Jacobi identities for $\star$ and $\star^{\prime}$-commutators then lead to $k[\Pi, \Lambda]=0$.

Lemma 5.10. The two Poisson bivectors $\Pi$ and $\Lambda$ are not compatible. 
Proof. The Schouten bracket is

$$
\begin{aligned}
{[\Pi, \Lambda] } & =2 \frac{\partial}{\partial \xi_{1}} \wedge \frac{\partial}{\partial \xi_{2}} \wedge\left(\xi_{1} \frac{\partial}{\partial x^{1}}+\xi_{2} \frac{\partial}{\partial x^{2}}\right) \\
& =2 \Lambda \wedge \frac{\mathrm{G}}{\mathrm{R}}
\end{aligned}
$$

where $\mathrm{G}$ and $\mathrm{R}$ are as in (3.5) and (3.4). This expression does not vanish.

Thus, the constant $k$ in the above formula has to vanish. This completes the proof of Part (iii).

Theorem [5.7 is proved.

Lemmas 5.85 .10 can be summarized as the following

Proposition 5.11. The second $\mathfrak{G}$-invariant Hochschild cohomology space is

$$
\mathrm{HH}_{\mathfrak{G}}^{2}(\mathcal{S}(M) ; \mathcal{S}(M))= \begin{cases}\mathbb{R}^{2}, & \text { in the conformal case for } n=2 \\ \mathbb{R}, & \text { otherwise }\end{cases}
$$

and the cup product in the first instance is non-zero.

This result could have been derived from Kontsevich's [34 or Fedosov's [27] classification of equivalence classes of deformations.

Remark 5.12. Theorem 5.7 does not guarantee uniqueness of a star-product but of a class of $\mathfrak{G}$-invariant star-products. Together with Propositions 3.1 and 3.2 this leads to an explicit description of all $\mathfrak{G}$-invariant star-products. Indeed, they are all obtained from the $\mathfrak{G}$-canonical homogeneous star-product by the equivalence (2.5) and reparametrization (2.6); the equivalence map $\Phi$ is given in terms of the $\mathfrak{G}$ invariant operators $\mathcal{E}$ in the projective case and $\mathcal{E}$ and $\mathrm{R}_{0}$ in the conformal case.

\subsection{Uniqueness up to $\mathfrak{G}$-equivalence and reparametrization, $\mathfrak{G}$-covariance and homogeneity}

In this section we compare our uniqueness theorems with those obtained for the Moyal star-product in 33. The Moyal star-product is the unique, up to reparametrization, $\left(\operatorname{Sp}(2 n, \mathbb{R}) \ltimes \mathbb{R}^{2 n}\right)$-invariant star-product on $\mathbb{R}^{2 n}$. It was also proved that it is uniquely selected within its reparametrization class by furthermore requiring its 
covariance. The $\left(\operatorname{Sp}(2 n, \mathbb{R}) \ltimes \mathbb{R}^{2 n}\right)$-equivalence class of the Moyal star-product has a single element since the $\left(\operatorname{Sp}(2 n, \mathbb{R}) \ltimes \mathbb{R}^{2 n}\right)$-commutant in $\operatorname{End}\left(C^{\infty}\left(\mathbb{R}^{2 n}\right)\right)$ is trivial so that there are no non-zero invariant Hochschild 2-coboundaries.

One may wonder if in our present setting $\mathfrak{G}$-covariance plays a similar role, namely, that of an extra condition that selects the canonical $\mathfrak{G}$-invariant star-product of Section 5.1 within its reparametrization and $\mathfrak{G}$-equivalence classes described in Section 5.2. The answer is negative; however we have

Proposition 5.13. If two $\mathfrak{G}$-invariant and $\mathfrak{G}$-covariant star-products on $\mathcal{S}(M)$ are equivalent up to reparametrization, then they coincide.

Proof. Let $\star$ and $\star^{\prime}$ be two $\mathfrak{G}$-invariant and $\mathfrak{G}$-covariant star-products on $\mathcal{S}(M)$ belonging to the same reparametrization class. Their $\mathfrak{G}$-covariance translates into $(\operatorname{see}(2.2))$ :

$$
J_{X} \star J_{Y}-J_{Y} \star J_{X}=i \hbar\left\{J_{X}, J_{Y}\right\}=J_{X} \star^{\prime} J_{Y}-J_{Y} \star^{\prime} J_{X}
$$

for all $X, Y \in \mathfrak{g}$. On the other hand reparametrization equivalence means that there exist a formal power series (2.6) such that

$$
F \star^{\prime} G=\sum_{r \geq 0}(i \hbar)^{r} B_{r}^{\prime}(F, G)=\sum_{r \geq 0}(\mu(i \hbar))^{r} B_{r}(F, G) .
$$

Using this equation, one rewrites the right hand side of (5.9) in terms of $\star$, with $\mu(i \hbar)$ as deformation parameter. Now, using the left hand side of (5.9) one gets $\mu(i \hbar)=i \hbar$, from which the conclusion follows.

An analog of the above statement, where the reparametrization equivalence is replaced by $\mathfrak{G}$-equivalence, does not hold. Indeed, one shows using an argument similar to the one in the above proof, that two $\mathfrak{G}$-invariant and $\mathfrak{G}$-covariant starproducts on $\mathcal{S}(M)$ in the same $\mathfrak{G}$-equivalence class, do not necessarily coincide. So, covariance does not play the same role for $\mathfrak{G}$ as it does for $\operatorname{Sp}(2 n, \mathbb{R}) \ltimes \mathbb{R}^{2 n}$. However, a simple verification shows that, for the Moyal star-product, homogeneity has exactly the same effect as $\left(\operatorname{Sp}(2 n, \mathbb{R}) \ltimes \mathbb{R}^{2 n}\right)$-covariance. Hence, the $\mathfrak{G}$-canonical and the Moyal star-products are uniquely determined by two simple conditions, namely, invariance and homogeneity. 


\section{Explicit formula for the projectively-invariant star-product}

In this section we compute the explicit formula of the canonical homogeneous projectively-invariant star-product. This solves a problem raised in [2].

Projective invariance will be dealt with in two stages. We first consider invariance with respect to an affine $\operatorname{subgroup} \operatorname{Aff}(n, \mathbb{R})$ of $\operatorname{SL}(n+1, \mathbb{R})$ and determine the affine-invariant bilinear operators on $\mathcal{S}\left(\mathbb{R P}^{n}\right)$. Those will be used to write down an Ansatz for the star-product we are looking for. We will then enforce full projective invariance by further demanding that inversions preserve the star-product. This will give rise to the equations (6.11) and (6.12) below. Another system of equations will arise from the associativity requirement (see (6.14) ). The unique solution of the complete system of equations will be given explicitly at the end of this section.

\subsection{Autonomous derivation from the invariance principle}

We need to classify the bilinear $\operatorname{Aff}(n, \mathbb{R})$-invariant differential operators on $\mathcal{S}\left(\mathbb{R}^{n}\right)$. For that purpose, let us resort to the natural isomorphism

$$
\mathcal{S}\left(\mathbb{R}^{n}\right) \otimes \mathcal{S}\left(\mathbb{R}^{n}\right) \cong \mathcal{S}\left(\mathbb{R}^{2 n}\right)
$$

and denote by $(x, \xi, y, \eta)$ the natural coordinate system on $T^{*} \mathbb{R}^{n} \times T^{*} \mathbb{R}^{n}$. The operators of divergence with respect to the first and the second arguments

$$
\mathrm{D}_{x \xi}(F, G)=D(F) G, \quad \mathrm{D}_{y \eta}(F, G)=F D(G),
$$

where $D$ is as in (3.3), and the operators of contraction

$$
\begin{aligned}
\mathrm{D}_{x \eta}(F, G) & =\left.\frac{\partial}{\partial x^{i}} \frac{\partial}{\partial \eta_{i}} F(\xi, x) G(\eta, y)\right|_{\eta=\xi, y=x}, \\
\mathrm{D}_{y \xi}(F, G) & =\left.\frac{\partial}{\partial y^{i}} \frac{\partial}{\partial \xi_{i}} F(\xi, x) G(\eta, y)\right|_{\eta=\xi, y=x}
\end{aligned}
$$

are obviously $\operatorname{Aff}(n, \mathbb{R})$-invariant differential operators. Restricting ourselves to homogeneous components, we get the following

Proposition 6.1. Every bilinear differential operator

$$
\mathcal{S}_{k}\left(\mathbb{R}^{n}\right) \otimes \mathcal{S}_{\ell}\left(\mathbb{R}^{n}\right) \rightarrow \mathcal{S}_{m}\left(\mathbb{R}^{n}\right)
$$


invariant with respect to the action of $\operatorname{Aff}(n, \mathbb{R})$, is a homogeneous polynomial in $\mathrm{D}_{\xi x}, \mathrm{D}_{\xi y}, \mathrm{D}_{\eta x}$ and $\mathrm{D}_{\eta y}$ of degree $k+\ell-m$.

This enables us to write the most general $\operatorname{Aff}(n, \mathbb{R})$-invariant bilinear operation $\mathcal{S}\left(\mathbb{R}^{n}\right) \otimes \mathcal{S}\left(\mathbb{R}^{n}\right) \rightarrow \mathcal{S}\left(\mathbb{R}^{n}\right)[\hbar]$. According to Theorem 5.7 we will express it as a termwise homogeneous formal series which, when restricted to $\mathcal{S}_{k}\left(\mathbb{R}^{n}\right) \otimes \mathcal{S}_{\ell}\left(\mathbb{R}^{n}\right)$, takes the form

$$
F \star G=\sum_{r=0}^{\infty}(i \hbar)^{r} B_{r}^{k, \ell}(F, G)
$$

where $B_{r}^{k, \ell}$ is a bidifferential operator, homogeneous of degree $r$ in $\mathrm{D}_{\xi x}, \mathrm{D}_{\xi y}, \mathrm{D}_{\eta x}, \mathrm{D}_{\eta y}$, viz

$$
B_{r}^{k, \ell}(F, G)(\xi, x)=\left.\sum_{\alpha+\beta+\gamma+\delta=r} B_{\alpha, \beta, \gamma, \delta}^{k, \ell} \mathrm{D}_{\xi y}^{\alpha} \mathrm{D}_{\eta x}^{\beta} \mathrm{D}_{\xi x}^{\gamma} \mathrm{D}_{\eta y}^{\delta} F(\xi, x) G(\eta, y)\right|_{\eta=\xi, y=x}
$$

with constant coefficients $B_{\alpha, \beta, \gamma, \delta}^{k, \ell}$.

Since we seek a star-product, we have to impose

$$
B_{0,0,0,0}^{k, \ell}=1
$$

and

$$
B_{1,0,0,0}^{k, \ell}=-B_{0,1,0,0}^{k, \ell}=\frac{1}{2}
$$

in order to get the multiplication and Poisson bracket as the first two terms as in equation (1.1).

Expressions (6.6) and (6.7) constitute our Ansatz for an $\operatorname{SL}(n+1, \mathbb{R})$-invariant star-product on $T^{*} \mathbb{R} \mathrm{P}^{n}$. It now remains to impose to the operation (6.6) the following conditions: (i) invariance with respect to inversions, and (ii) associativity.

\subsection{Projective invariance}

Let $X_{i}=x^{i} x^{j} \partial_{x^{j}}$ be the $i$-th generator of inversions. Denote by

$$
L_{X_{i}}=x^{i} x^{j} \frac{\partial}{\partial x^{j}}-x^{j} \xi_{j} \frac{\partial}{\partial \xi_{i}}-x^{i} \xi_{j} \frac{\partial}{\partial \xi_{j}}+y^{i} y^{j} \frac{\partial}{\partial y^{j}}-y^{j} \eta_{j} \frac{\partial}{\partial \eta_{i}}-y^{i} \eta_{j} \frac{\partial}{\partial \eta_{j}}
$$

its canonical lift to $T^{*}\left(\mathbb{R}^{2 n}\right)$. 
Invariance with respect to inversions translates into the following equations

$$
\left.\sum_{\alpha+\beta+\gamma+\delta=r} B_{\alpha, \beta, \gamma, \delta}^{k, \ell}\left[L_{X_{i}}, \mathrm{D}_{\xi y}^{\alpha} \mathrm{D}_{\eta x}^{\beta} \mathrm{D}_{\xi x}^{\gamma} \mathrm{D}_{\eta y}^{\delta}\right]\right|_{\eta=\xi, y=x}=0
$$

at each order $r \in \mathbb{N}$. The latter yield the following system of equations

$$
\begin{aligned}
& (\alpha+1)(\alpha+\delta-\ell) B_{\alpha+1, \beta, \gamma, \delta}^{k, \ell}+(\beta+1)(\beta+\delta-\ell) B_{\alpha, \beta+1, \gamma, \delta}^{k, \ell}= \\
& (\gamma+1)(n+2 k-\gamma-1) B_{\alpha, \beta, \gamma+1, \delta}^{k, \ell}+(\alpha+1)(\beta+1) B_{\alpha+1, \beta+1, \gamma, \delta-1}^{k, \ell}
\end{aligned}
$$

and

$$
\begin{aligned}
& (\beta+1)(\beta+\gamma-k) B_{\alpha, \beta+1, \gamma, \delta}^{k, \ell}+(\alpha+1)(\alpha+\gamma-k) B_{\alpha+1, \beta, \gamma, \delta}^{k, \ell}= \\
& (\delta+1)(n+2 \ell-\delta-1) B_{\alpha, \beta, \gamma, \delta+1}^{k, \ell}+(\alpha+1)(\beta+1) B_{\alpha+1, \beta+1, \gamma-1, \delta}^{k, \ell} .
\end{aligned}
$$

\subsection{Associativity}

If $F \in \mathcal{S}_{k}\left(\mathbb{R}^{n}\right), G \in \mathcal{S}_{\ell}\left(\mathbb{R}^{n}\right)$, and $H \in \mathcal{S}_{m}\left(\mathbb{R}^{n}\right)$ the associativity condition takes the form

$$
\sum_{j=0}^{r} B_{r-j}^{k, \ell+m-j}\left(F, B_{j}^{\ell, m}(G, H)\right)=\sum_{j=0}^{r} B_{r-j}^{k+\ell-j, m}\left(B_{j}^{k, \ell}(F, G), H\right)
$$

for all $r \in \mathbb{N}$. Equation (6.13) then reads

$$
\begin{aligned}
& \sum_{j=0}^{r} \sum_{\alpha+\beta+\gamma+\delta=r-j} B_{\alpha, \beta, \gamma, \delta}^{k, \ell+m-j}\left(\mathrm{D}_{\xi y}+\mathrm{D}_{\xi z}\right)^{\alpha}\left(\mathrm{D}_{\eta x}+\mathrm{D}_{\zeta x}\right)^{\beta} \times \\
& \mathrm{D}_{\xi x}^{\gamma}\left(\mathrm{D}_{\eta y}+\mathrm{D}_{\eta z}+\mathrm{D}_{\zeta y}+\mathrm{D}_{\zeta z}\right)^{\delta} \times \\
& \sum_{\alpha^{\prime}+\beta^{\prime}+\gamma^{\prime}+\delta^{\prime}=j} B_{\alpha^{\prime}, \beta^{\prime}, \gamma^{\prime}, \delta^{\prime}}^{\ell, m} \mathrm{D}_{\eta z}^{\alpha^{\prime}} \mathrm{D}_{\zeta y}^{\beta^{\prime}} \mathrm{D}_{\eta y}^{\gamma^{\prime}} \mathrm{D}_{\zeta z}^{\delta^{\prime}}= \\
& \sum_{j=0}^{r} \sum_{\alpha+\beta+\gamma+\delta=r-j} B_{\alpha, \beta, \gamma, \delta}^{k+\ell-j, m}\left(\mathrm{D}_{\xi z}+\mathrm{D}_{\eta z}\right)^{\alpha}\left(\mathrm{D}_{\zeta x}+\mathrm{D}_{\zeta y}\right)^{\beta} \times \\
& \left(\mathrm{D}_{\xi x}+\mathrm{D}_{\xi y}+\mathrm{D}_{\eta x}+\mathrm{D}_{\eta y}\right)^{\gamma} \mathrm{D}_{\zeta z}^{\delta} \times \\
& \sum_{\alpha^{\prime}+\beta^{\prime}+\gamma^{\prime}+\delta^{\prime}=j} B_{\alpha^{\prime}, \beta^{\prime}, \gamma^{\prime}, \delta^{\prime}}^{k,} \mathrm{D}_{\xi y}^{\alpha^{\prime}} \mathrm{D}_{\eta x}^{\beta^{\prime}} \mathrm{D}_{\xi x}^{\gamma^{\prime}} \mathrm{D}_{\eta y}^{\delta^{\prime}} .
\end{aligned}
$$

\subsection{Explicit solution of the system}

We solve the system of equations (6.11), (6.12) and (6.14), by first determining the components $B_{\alpha, \beta, 0,0}^{k, \ell}$, then $B_{\alpha, \beta, \gamma, 0}^{k, \ell}$ and, finally, the full expression $B_{\alpha, \beta, \gamma, \delta}^{k, \ell}$. 


\subsubsection{First stage}

Identifying in the associativity equation (6.14) the coefficients of the monomials $\mathrm{D}_{\xi z}^{r-j} \mathrm{D}_{\zeta x}^{j}$, one readily finds $B_{r-j, j, 0,0}^{k, \ell+m}=B_{r-j, j, 0,0}^{k+\ell, m}$. Thus, $B_{\alpha, \beta, 0,0}^{k, \ell}$ depends only on $k+\ell$; we write

$$
B_{\alpha, \beta, 0,0}^{k, \ell}=C_{\alpha, \beta}(k+\ell) .
$$

Using again (6.14), we identify the coefficients of the monomials $\mathrm{D}_{\xi z}^{r-j-1} \mathrm{D}_{\zeta x}^{j} \mathrm{D}_{\eta x}$ and $\mathrm{D}_{\xi z}^{r-j-1} \mathrm{D}_{\zeta x}^{j} \mathrm{D}_{\xi y}$, respectively, to get the following system

$$
\begin{aligned}
(j+1) B_{r-j-1, j+1,0,0}^{k, \ell+m} & =B_{r-j-1, j, 1,0}^{k+\ell, m}+B_{r-j-1, j, 0,0}^{k+\ell, m} B_{0,1,0,0}^{k, \ell}, \\
(r-j) B_{r-j, j, 0,0}^{k, \ell+m} & =B_{r-j-1, j, 1,0}^{k+\ell, m}+B_{r-j-1, j, 0,0}^{k+\ell-1, m} B_{1,0,0,0}^{k, \ell} .
\end{aligned}
$$

Resorting to the invariance equation (6.11) for $\alpha=r-j-1, \beta=j$, and $\gamma=\delta=0$, we obtain the supplementary equation

$(r-j)(r-j-\ell-1) B_{r-j, j, 0,0}^{k, \ell}+(j+1)(j-\ell) B_{r-j-1, j+1,0,0}^{k, \ell}-(n+2 k-1) B_{r-j-1, j, 1,0}^{k, \ell}=0$.

The previous three equations together with (6.9) and (6.15) imply

$$
(r-j)(r-n-2 k) C_{r-j, j}(k)+\frac{1}{2}(n+2 k-2 j-1) C_{r-j-1, j}(k-1)=0 .
$$

The latter equation, supplemented with (6.8), yields then

$$
B_{\alpha, \beta, 0,0}^{k, \ell}=\frac{(-1)^{\beta}}{(\alpha+\beta) !} \frac{\left(\begin{array}{c}
\frac{1}{2}(n-1)+k+\ell-\beta \\
\alpha
\end{array}\right)\left(\begin{array}{c}
\frac{1}{2}(n-1)+k+\ell-\alpha \\
\beta
\end{array}\right)}{\left(\begin{array}{c}
n+2 k+2 \ell-\alpha-\beta \\
\alpha+\beta
\end{array}\right)}
$$

\subsubsection{Second stage}

Here we only use the first invariance equation (6.11) with $\delta=0$. Long but straightforward calculations lead to

$$
B_{\alpha, \beta, \gamma, 0}^{k, \ell}=\frac{1}{\gamma !(n+2 k-\gamma)_{\gamma}} \sum_{r+s=\gamma}\left(\begin{array}{l}
\gamma \\
r
\end{array}\right)(\alpha+1)_{r}(\beta+1)_{s}(\alpha-\ell)_{r}(\beta-\ell)_{s} B_{\alpha+r, \beta+s, 0,0}^{k, \ell}
$$

where the last term is as in (6.16). 


\subsubsection{Last stage}

A reverse iterative computation on $\delta$ using the second invariance equation (6.12) finally leads to the sought for result

$$
\begin{aligned}
B_{\alpha, \beta, \gamma, \delta}^{k, \ell}= & \frac{1}{\delta !(n+2 \ell-\delta)_{\delta}} \sum_{r+s+t=\delta}(-1)^{s}\left(\begin{array}{c}
\delta \\
r, s, t
\end{array}\right) \times \\
& (\alpha+1)_{r}(\alpha+1)_{s}(\beta+1)_{s}(\beta+1)_{t}(\alpha+\gamma-k)_{r}(\beta+\gamma-k)_{t} \times \\
& B_{\alpha+r+s, \beta+s+t, \gamma-s, 0}^{k, \ell}
\end{aligned}
$$

where the first line contains the trinomial coefficient and the last one is given by (6.17).

\subsection{Symmetry condition}

Proposition 6.2. The symmetry condition C2 translates for the Ansatz (6.6)- 6.7) into

$$
B_{\alpha, \beta, \gamma, \delta}^{k, \ell}=(-1)^{\alpha+\beta+\gamma+\delta} B_{\beta, \alpha, \delta, \gamma}^{\ell, k} .
$$

Proof. If $F \in \mathcal{S}_{k}\left(\mathbb{R}^{n}\right)$, and $G \in \mathcal{S}_{\ell}\left(\mathbb{R}^{n}\right)$, we immediately get from Condition C2 that

$$
B_{r}^{k, \ell}(F, G)=(-1)^{r} B_{r}^{\ell, k}(G, F)
$$

Then, a change of dummy variables in (6.7) completes the proof.

It turns out that our star-product given by (6.6), (6.7) and (6.18) automatically satisfies the symmetry condition (6.19). Although this is not transparent from the expression (6.18), it is however a direct consequence of Proposition 4.4 and Theorem 5.1 .

\section{Conclusion, discussion and outlook}

In this work we have proved the existence and uniqueness of a canonical $\mathfrak{G}$-invariant star-product on $T^{*} M$ for $\mathfrak{G}=\mathrm{SL}(n+1, \mathbb{R})\left(\right.$ resp. $\mathfrak{G}=\mathrm{SO}_{0}(p+1, q+1)$ and $M=\mathbb{R} \mathrm{P}^{n}$ (resp. $\left.\left(S^{p} \times S^{q}\right) / \mathbb{Z}_{2}\right)$. We have, moreover, given an explicit formula for the canonical projectively invariant star-product. For both geometries, the canonical star-product 
so obtained is symmetric, homogeneous, strongly $\mathfrak{G}$-invariant (hence $\mathfrak{G}$-covariant), but not differential. These properties, except for the last one, are shared with the Moyal star-product on $\mathbb{R}^{2 n}$.

Theorem 5.1 shows that the homogeneity condition supplementing $\mathfrak{G}$-invariance uniquely determines the canonical $\mathfrak{G}$-invariant star-product on $\mathcal{S}(M)$. Likewise, the Moyal star-product is also uniquely specified by $\left(\operatorname{Sp}(2 n, \mathbb{R}) \ltimes \mathbb{R}^{2 n}\right)$-invariance and homogeneity. This allows us to draw a parallel between our canonical $\mathfrak{G}$-invariant star-product and Moyal's, namely, they are uniquely determined by the same two simple conditions : invariance and homogeneity. Of course, this parallel is far from complete, since, for instance, $\mathfrak{G}$ and $\operatorname{Sp}(2 n, \mathbb{R}) \ltimes \mathbb{R}^{2 n}$ do not have the same geometric status; the action of the former on $T^{*} M$ is lifted from that on $M$, which is not the case for the latter.

Furthermore, it is clear that, for the projective and the conformal cases, there is no $\mathfrak{G}$-invariant (symplectic) connection on $T^{*} M$, since $\mathfrak{G}$ does not act on the bundle of linear frames of $T^{*} M$. Hence, no Fedosov [26] canonical $\mathfrak{G}$-invariant starproduct can be constructed. Besides, Fedosov's construction would have led to a star-product given by bidifferential operators.

The generalization of the existence and uniqueness theorems for projectively/conformally invariant star-products on $T^{*} M$ in the case of a non-flat projective/conformal connection on $M$ remains an open problem. In a recent work [6], Bordemann has taken a significant step in this new direction, by investigating the projectively equivariant quantization on a cotangent bundle of a manifold with a non flat projective structure (see also [21] and [7]). Note also, that since the canonical star-products studied in this work may be considered as the projective/conformal analogs of the Moyal star-product, they may play a similar role as the latter in a construction à la Fedosov of a star-product on a symplectic manifold with a Cartan projective/conformal symplectic connection.

In the case $n \geq 2$, let us mention that the explicit form of the conformally invariant star-product is, so far, out of reach. This was already the situation for the conformally equivariant quantization map [23] (see also [21]).

In the conformal case with $n=2$, Theorem [5.7 holds for star-products of the form (1.1) with the standard Poisson bracket on $T^{*} M$ as first-order term. However, one could easily construct, in this case, another $\mathfrak{G}$-invariant star-product with the 
Poisson bracket (5.4) as first-order term. It would be interesting to give a physical status to this second, somewhat "exotic", star-product.

In the case of dimension $n=1$, our results are related to earlier work by Cohen, Manin and Zagier [12. The projective and the conformal algebras are, in this case, isomorphic to $\operatorname{sl}(2, \mathbb{R})$. Moreover, the canonical projectively and conformally invariant star-products coincide by uniqueness and thus the explicit formulæ given in Section 6.4 correspond to the one obtained in [12] for $\lambda=\frac{1}{2}$.

\section{References}

[1] D. Arnal, J.-C. Cortet, P. Molin, G. Pinczon, Covariance and geometrical invariance in star-quantization, J. Math. Phys. 24 (1983) 276-283.

[2] A. Astashkevich, R. Brylinski, Non-Local Equivariant Star Product on the Minimal Nilpotent Orbit, to appear in Advances in Math, math.QA/0010257 v2.

[3] F. Bayen, M. Flato, C. Fronsdal, A. Lichnerowicz, D. Sternheimer, Deformation theory and quantization. I. Deformations of symplectic structures, Ann. Physics 111:1 (1978) 61-110.

[4] M. Bertelson, P. Bieliavsky, S. Gutt, Parametrizing equivalence classes of invariant star products, Lett. Math. Phys. 46:4 (1998) 339-345.

[5] F. Boniver, P.B.A. Lecomte, A remark about the Lie algebra of infinitesimal conformal transformations of the Euclidean space, Bull. London Math. Soc. 32:3 (2000), 263-266.

[6] M. Bordemann, Sur l'existence d'une prescription d'ordre naturelle projectivement invariante, math.DG/0208171.

[7] S. Bouarroudj, Projectively equivariant quantization map, Lett. Math. Phys. $51: 4(2000)$ 265-274.

[8] R. Brylinski, Equivariant Deformation Quantization for the Cotangent Bundle of a Flag Manifold, Ann. Inst. Fourier 52:3 (2002) 881-897. 
[9] R. Brylinski, Non-Locality of Equivariant Star Products on $T^{*}\left(R P^{n}\right)$, Lett. Math. Phys. 58:1 (2001) 21-28.

[10] M. Cahen, S. Gutt, M. De Wilde, Local cohomology of the algebra of $C^{\infty}$ functions on a connected manifold, Lett. Math. Phys. 4:3 (1980) 157-167.

[11] A. Cattaneo, G. Felder, A path integral approach to the Kontsevich quantization formula, Comm. Math. Phys. 212:3 (2000) 591-611.

[12] P. Cohen, Yu. Manin, D. Zagier, Automorphic pseudodifferential operators, Algebraic aspects of integrable systems, 17-47, Progr. Nonlinear Differential Equations Appl., 26, Birkhäuser Boston, Boston, MA, 1997.

[13] A. Connes, Noncommutative differential geometry, Inst. Hautes Etudes Sci. Publ. Math. No. 62 (1985) 257-360.

[14] P. Deligne, Déformations de l'algèbre des fonctions d'une variété symplectique: comparaison entre Fedosov et De Wilde, Lecomte, Selecta Math. (N.S.) 1:4 (1995) 667-697.

[15] M. De Wilde, P. Lecomte, Star-products on cotangent bundles, Lett. Math. Phys. 7:3 (1983) 235-241.

[16] M. De Wilde, P. Lecomte, Existence of star-products and of formal deformations of the Poisson Lie algebra of arbitrary symplectic manifolds, Lett. Math. Phys. 7:6 (1983) 487-496.

[17] M. De Wilde, P. Lecomte, An homotopy formula for the Hochschild cohomology, Compositio Math. 96:1 (1995) 99-109.

[18] G. Dito, D. Sternheimer, Deformation quantization: genesis, developments and metamorphoses, IRMA Lectures in math. Theoret. Phys. 1, Walter de Gruyter, Berlin (2002) 9-54.

[19] B. A. Dubrovin, A. T. Fomenko, S. P. Novikov, Modern geometry-methods and applications. Part I. Graduate Texts in Mathematics, 93. Springer-Verlag, New York, 1992. 
[20] C. Duval, V. Ovsienko, Space of second order linear differential operators as a module over the Lie algebra of vector fields, Advances in Math. 132:2 (1997) 316-333.

[21] C. Duval, V. Ovsienko, Conformally equivariant quantum Hamiltonians, Selecta Math. (N.S.) 7:3 (2001) 291-320.

[22] C. Duval, V. Ovsienko, Projectively equivariant quantization and symbol calculus: noncommutative hypergeometric functions, Lett. Math. Phys. 57:1 (2001) 61-67.

[23] C. Duval, P. Lecomte, V. Ovsienko, Conformally equivariant quantization: existence and uniqueness, Ann. Inst. Fourier. 49:6 (1999) 1999-2029.

[24] B.V. Fedosov, Formal quantization, Some topics of modern mathematics and their applications to problems of mathematical physics (in Russian), 129-136, Moscow (1985).

[25] B.V. Fedosov, A simple geometrical construction of deformation quantization, J. Diff. Geom. 40:2 (1994) 213-238.

[26] B.V. Fedosov, Non-abelian reduction in deformation quantization, Lett. Math. Phys. 43:2 (1998) 137-154.

[27] B.V. Fedosov, Deformation quantization and index theory. Mathematical Topics, 9 Akademie Verlag, Berlin, 1996.

[28] M. Gerstenhaber, On the deformation of rings and algebras, Ann. of Math. 79:2 (1964) 59-103.

[29] R. Goodman, N. Wallach, Representations and invariants of the classical groups. Encyclopedia of Mathematics and its Applications, 68, Cambridge University Press, Cambridge, 1998.

[30] R.L. Graham, D.E. Knuth, O. Patashnik, Concrete Mathematics, AddisonWesley, 1992.

[31] H.J. Gronewold, On the principles of elementary quantum mechanics, Physica 12 (1946) 405-460. 
[32] S. Gutt, Variations on deformation quantization, Math. Phys. Stud., 21, Kluwer Acad. Publ., Dordrecht, 2000, 217-254.

[33] S. Gutt, Contribution à l'étude des espaces symplectiques homogènes, Acad. Roy. Belg. Cl. Sci. Mém. Collect. $8^{\circ}$ (2) 44:6 (1983).

[34] M. Kontsevich, Deformation quantization of Poisson manifolds I, q-alg/9709040.

[35] P.B.A. Lecomte, Classification projective des espaces d'opérateurs différentiels agissant sur les densités, C. R. Acad. Sci. Paris Sér. I Math. 328:4 (1999), $287-290$.

[36] P.B.A. Lecomte, On the cohomology of $\operatorname{sl}(m+1, \mathbb{R})$ acting on differential operators and $\mathrm{sl}(m+1, \mathbb{R})$-equivariant symbol, Indag. Math., N.S., 11:1 (2000) $95-114$.

[37] P.B.A. Lecomte and V. Ovsienko, Projectively invariant symbol calculus, Lett. Math. Phys. 49:3 (1999), 173-196.

[38] A. Lichnerowicz, Déformations d'algèbres associées à une variété symplectique (les $*_{\nu}$-produits), Ann. Inst. Fourier 32:1 (1982) 157-209.

[39] J.E. Moyal, Quantum mechanics as a statistical theory, Proc. Cambridge Philos. Soc. 45 (1949) 99-124.

[40] F. Nadaud, On continuous and differential Hochschild cohomology, Lett. Math. Phys. 47:1 (1999) 85-95.

[41] H. Omori, Y. Maeda, A. Yoshioka, Weyl manifolds and deformation quantization, Adv. Math. 85:2 (1991) 224-255.

[42] J. Peetre, Une caractérisation abstraite des opérateurs différentiels, Math. Scand. 7 (1959), 211-218 and 8 (1960), 116-120.

[43] D.E. Tamarkin, Another proof of M. Kontsevich formality theorem, math.QA/9803025. 
[44] J. Vey, Déformation du crochet de Poisson sur une variété symplectique, Comment. Math. Helv. 50:4 (1975) 421-454.

[45] A. Weinstein, Deformation quantization, Séminaire Bourbaki, 1993/94, Astérisque 227 (1995), 389-409.

[46] H. Weyl, The Classical Groups, Princeton University Press, 1946. 